\title{
What is the Impact of Abnormal Amniotic Fluid Volumes on Perinatal Outcomes in Normal Compared with At-Risk Pregnancies?
}

This article was published in the following Dove Press journal: International Journal of Women's Health

\author{
Pamela M Simmons ${ }^{1,2}$ \\ Julie R Whittington (1) \\ Sarah M Estrada ${ }^{3}$ \\ Songthip T Ounpraseuth ${ }^{4}$ \\ Kelsey L Shnaekel' \\ Kala B Slaton' \\ Everett F Magann (D) \\ 'University of Arkansas for Medical \\ Sciences, College of Medicine, \\ Department of Obstetrics and \\ Gynecology, Little Rock, AR, USA; \\ ${ }^{2}$ Women's Hospital, Department of \\ Obstetrics and Gynecology, Baton \\ Rouge, LA, USA; ${ }^{3}$ Madigan Army Medical \\ Center, Department of Obstetrics and \\ Gynecology, Joint Base Lewis-McChord, \\ WA, USA; ${ }^{4}$ University of Arkansas for \\ Medical Sciences, College of Public \\ Health, Department of Biostatistics, Little \\ Rock, AR, USA
}

Correspondence: Everett F Magann University of Arkansas for Medical Sciences, College of Medicine, Department of Obstetrics and Gynecology, 430I W. Markham St. Slot \# 518, Little Rock, AR 72205-7199, USA

Tel + I50I-686-8345

Fax + I50I-526-7820

Email efmagann@uams.edu
Objective: Assessing amniotic fluid volume is an integral part of obstetric practice. Data are sparse on at-risk pregnancy and amniotic fluid volumes. The aim of our study was to determine if there is a difference in perinatal outcomes based on complications of pregnancy and amniotic fluid volumes. We hypothesized that at-risk pregnancies with abnormal amniotic fluid volumes would have worse perinatal outcomes than normal pregnancies with abnormal amniotic fluid volumes.

Study Design: This retrospective cohort study evaluated both normal and at-risk singleton pregnancies with intact membranes on admission for delivery. Amniotic fluid volumes were estimated using both the amniotic fluid index (AFI) and single deepest pocket (SDP) techniques. All sonograms were performed by trained ultrasound technicians or obstetrician/gynecologists. We placed 3365 women into 6 separate groups (at-risk versus normal, then further stratified by oligohydramnios by SDP, normal fluid, or polyhydramnios by AFI). Results: At-risk pregnancies with normal fluid and at-risk pregnancies with polyhydramnios have significantly increased risk of neonatal intensive care unit (NICU) admission [OR 2.06 (95\% CI 1.63,2.60), OR 2.74 (95\% CI 1.54, 4.87)]. Birthweight is significantly higher in at-risk and normal pregnancies with polyhydramnios than those with normal pregnancies and normal fluid $(\mathrm{p}<0.0001)$. Birthweight is significantly lower in at-risk pregnancies with oligohydramnios $(p<0.0001)$. There were no significant differences in need for amnioinfusion in labor, variables or lates influencing delivery, meconium staining, or umbilical artery $\mathrm{pH}<7.1$.

Conclusion: Our study attempted to further define risk of adverse pregnancy outcomes by defining the pregnancy as normal or at-risk and amniotic fluid volumes. Contrary to our hypothesis, we did not find an increased risk of many of the adverse perinatal outcomes we studied amongst at-risk pregnancies with abnormal fluid. There was an increased risk of NICU admission associated with polyhydramnios in normal and at-risk pregnancies.

Keywords: amniotic fluid volume, obstetric ultrasound, neonatal outcomes, at-risk pregnancy

\section{Plain Language Summary}

Ultrasound estimates of amniotic fluid volume are used to manage pregnancies that are atrisk for adverse outcomes. Both high and low amniotic fluid volume have been associated with pregnancy complications. This study looked at normal pregnancies and higher risk pregnancies along with the amount of amniotic fluid each woman had prior to delivery. We expected that women with low or high amniotic fluid volumes would have increased rates of complications. We also expected that the higher risk pregnancies would have increased rates of complications. There were no significant differences in many of our selected markers of pregnancy complications (decelerations in fetal heart rate requiring influencing need for 
delivery, need for infusion of fluid to alleviate decelerations, low umbilical artery $\mathrm{pH}$ and meconium staining of amniotic fluid). Neonatal intensive care unit admission is increased in normal pregnancy with excess amniotic fluid and at-risk pregnancy with excess amniotic fluid. There is a continued need in obstetrics to risk stratify pregnancies in order to target interventions that reduce adverse pregnancy outcomes.

\section{Introduction}

The sonographic estimate of the amniotic fluid volume (AFV) is used as an indicator of fetal well-being and is an integral part of antenatal testing. ${ }^{1}$ It is postulated that an "at-risk" or abnormal pregnancy could affect the AFV of a pregnancy. This could be due to poor placental perfusion, which causes blood to shunt to the brain, heart, and adrenal glands at the expense of the rest of the fetal organ systems, resulting in decreased renal perfusion and oligohydramnios. ${ }^{2}$ Conversely, amniotic fluid can be increased in pregnancies complicated with diabetes or with macrosomia. ${ }^{3}$ Because of these associations, measurement of the AFV has become a vital part of the fetal surveillance techniques designed for pregnancies at risk for adverse perinatal outcomes. ${ }^{4}$ The two most commonly used surveillance tests are the biophysical profile and the modified biophysical profile, both of which require the assessment of amniotic fluid volume with the single deepest pocket (SDP) technique and/or the amniotic fluid index (AFI) to complete the antenatal testing.

A number of investigations have defined normal AFV across gestation in normal pregnancies. ${ }^{5-7}$ AFVs in at-risk pregnancies have been compared to the AFV normalities derived from low risk women, and the presence of normal amniotic fluid volumes is reassuring even in at-risk women. ${ }^{8}$ Very few investigations have evaluated the relationships between normal and abnormal AFVs in a large, single study of both normal and at-risk, non-anomalous singleton pregnancies with intact membranes. The purpose of this investigation is to evaluate the relationship of estimated AFV and outcomes in normal and at-risk pregnancies. Our objective was to determine if there is a difference in neonatal outcomes based upon at-risk classification of the pregnancy and amniotic fluid volumes (both AFI and/or SDP). We hypothesized that at-risk pregnancies with abnormal amniotic fluid volumes would have worse perinatal outcomes than normal pregnancies with abnormal amniotic fluid volumes.

\section{Materials and Methods}

This retrospective cohort study evaluated both normal and at-risk singleton pregnancies after admission to labor and delivery. The study was conducted at Madigan Army Medical Center and the University of Arkansas for Medical Sciences (UAMS). This study was approved by the Institutional Review Board of the University of Arkansas for Medical Sciences (study \#205741). The Investigational Review Board determined that informed consent was not required, as the study was retrospective in nature and patient medical record number was removed from the data set after obtaining data. This study was conducted in accordance with the Strengthening the Reporting of Observational Studies in Epidemiology (STROBE) guidelines. ${ }^{9}$

Women with multiples, suspected or confirmed aneuploidy, fetal anomalies, prelabor rupture of membranes, and those admitted with the anticipation of cesarean delivery were excluded from the study. A normal pregnancy was defined as subjects without medical conditions that could influence amniotic fluid volume. An "at-risk" pregnancy was defined as subjects with medical conditions that could influence amniotic fluid volume such as chronic hypertension, preeclampsia, pre- or gestational diabetes, collagen vascular disorders, renal disorders and chronic placental abruptions.

All women with intact membranes admitted to labor and delivery from July 2015 to December 2018 with the anticipation of spontaneous vaginal delivery at the University of Arkansas for Medical Sciences and Madigan Army Medical Center were evaluated for inclusion in the study. Once meeting inclusion criteria, data on maternal and perinatal outcomes, maternal demographics, medical history, and antepartum complications were evaluated. Intrapartum assessments included mode of delivery, reason for operative vaginal delivery or cesarean delivery if they occurred, and meconium staining of the amniotic fluid. Neonatal outcomes included gestational age at delivery, Apgar scores at 1 and 5 minutes, umbilical artery cord $\mathrm{pH}$, base excess, admission to the neonatal intensive care unit (NICU), and reason for that admission. The reasons for admission included prematurity, sepsis workup, chorioamnionitis, respiratory depression, hypoglycemia, meconium aspiration, respiratory morbidity, and perinatal death.

Amniotic fluid volumes were estimated using both the AFI and SDP techniques. All sonograms were performed by 
trained ultrasound technicians or obstetrician/gynecologists. For patients with more than one ultrasound, the most recent sonographic estimation of fluid prior to the delivery and before rupture of the membranes was used in the analysis. Amniotic fluid was classified as oligohydramnios, normal, or polyhydramnios. We defined oligohydramnios using the SDP measurement as this has been shown to be a superior method for assessing the AFV when amniotic fluid is low. Despite the fact that the predictability of the SDP and AFI are similar, the use of the AFI leads to more inductions of labor and more operative deliveries without an improvement in perinatal outcomes. ${ }^{10-12}$ The AFI was used to identify AFV as high instead of SDP. Although the AFI and SDP identify actual high volumes similarly, the AFI has a higher predictive value for identifying high volumes and the use of the SDP might lead to the over-diagnosis of high volumes leading to more interventions ${ }^{13}$ (Figure 1).

\section{Statistical Analysis}

We placed the patients into separate groups according to the following.

1. An "at-risk" pregnancy was defined as a pregnancy with any of the following maternal conditions which can be associated with abnormal amniotic fluid volumes: chronic hypertension, preeclampsia/ eclampsia, maternal diabetes, development of fetal viral infection, collagen vascular disease, chronic renal disease, chronic abruption.

2. Using the last observed assessment value, SDP < $2 \mathrm{~cm}$ was used to define oligohydramnios; AFI $>$ $24 \mathrm{~cm}$ was used to define polyhydramnios; normal otherwise

3. Based on 1 and 2 we defined 6 groups:
a. Normal + Normal fluid
b. Normal + Oligohydramnios
c. Normal + Polyhydramnios
d. At-Risk + Normal fluid
e. At-Risk + Oligohydramnios
f. At-Risk + Polyhydramnios

Initially, maternal characteristics were summarized using means \pm standard deviations or median (25th, 75th percentiles), as appropriate, for continuous variables and frequency (percentage) for categorical measures stratified by group. Additionally, one-way analysis of variance (ANOVA), Kruskal-Wallis test, or chi-square test was used to compare the maternal characteristics across the six groups. Adjustment for pairwise comparisons was done using Bonferroni correction. Unadjusted and multivariable logistic regression models were used to examine the association between the groups with dichotomous infant outcomes including: need for amnioinfusion during labor, variables influencing delivery, meconium staining, NICU admission, and umbilical artery $\mathrm{pH}<7.1$. We chose 7.1 as the $\mathrm{pH}$ cut off as it is the 2.5 percentile from a large retrospective database. ${ }^{14}$ Both unadjusted and adjusted odds ratios along with their respective 95\% confidence intervals (CI) are reported. For the continuous infant outcome measures such as birthweight and base excess, simple and multiple regression models were used to obtain least square mean difference along with the 95\% CIs. All analyses were performed using SAS 9.4 (SAS Institute, Cary, NC, USA) with a two-sided significance level of 0.05 .

\section{Results}

Information on the 3365 patients who met inclusion criteria for analysis were collected, and were divided into six groups: 2198 (65.3\%) "Normal + Normal Fluid", 80 (2.4\%) "Normal + Oligohydramnios", $74 \quad(2.2 \%)$ "Normal + Polyhydramnios", 917 (27.3\%) "At-Risk + Normal Fluid”, $21(0.6 \%)$ "At-Risk + Oligohydramnios", and 75 (2.2\%) "AtRisk Polyhydramnios". There was a statistical difference in maternal age across the six groups with the youngest group being patients from the Normal + Oligohydramnios cohort $(24.7 \pm 6.3)$ while the At-Risk + Normal Fluid group was the oldest on average $(29.5 \pm 6.1)$. The Normal + Polyhydramnios group had the smallest average BMI during the first visit $(27.9 \pm 5.5)$ while the At-Risk + Oligohydramnios had the largest average BMI (35.4 \pm 8.2$)$. More specifically, the average BMI during the first visit for patients in the Normal + Polyhydramnios group was significantly lower compared to those in any of the "At-Risk" cohorts $(\mathrm{p}<0.05)$. Additionally, those patients in the Normal + Normal Fluid had significantly lower BMI compared to patients in both the At-Risk + Oligohydramnios and At-Risk + Polyhydramnios $(\mathrm{p}<0.05)$. There were fewer Caucasian patients in the Normal + Oligohydramnios and At-Risk + Oligohydramnios groups compared to the Normal + Polyhydramnios and At-Risk + Polyhydramnios groups. There were no differences in gravidity or parity across the six groups (Table 1).

For the logistic regression analyses, the Normal + Normal Fluid was used as the reference group. Among the binary 


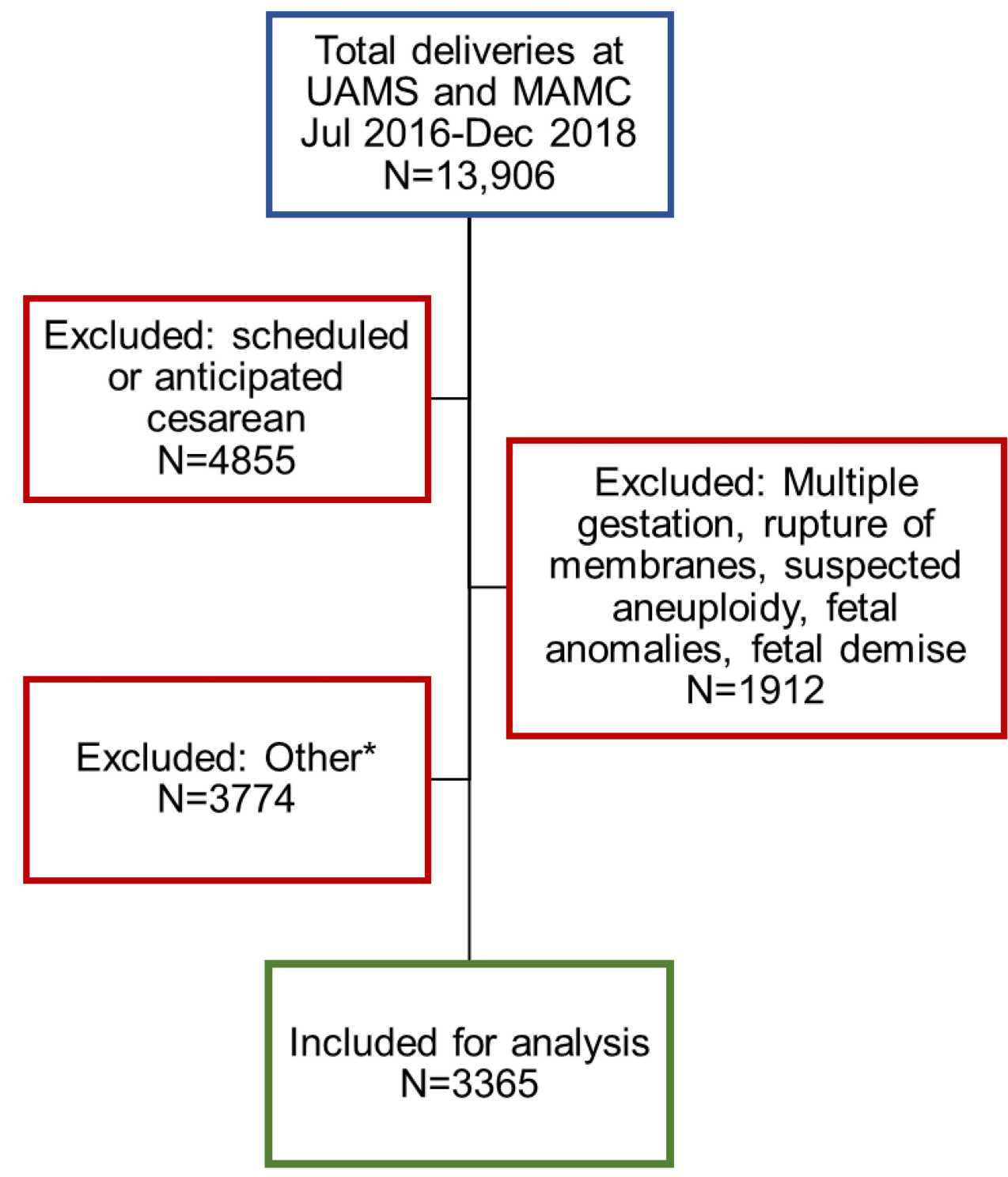

*Missing data from fetus or mother, $<20$ weeks gestation

Figure I Study flow.

infant outcome measures, only NICU admission was statistically significant in both the unadjusted and adjusted models (Table 2, Supplemental Table 1). The multivariable logistic regression model included maternal age, race, parity, gravidity, BMI at first visit, and estimated gestational age at the last assessment as covariates (Table 2). Infants born to mothers in the At-Risk + Normal Fluid had adjusted an OR $=2.06$ (95\% CI: $1.63,2.60)$ of requiring a NICU admission compared to those born to mothers in the Normal + Normal Fluid group. In other words, the likelihood of a NICU admission for infants born to mothers in the At-Risk + Normal
Fluid was $106 \%$ higher compared to those born to mothers in the Normal + Normal Fluid group. Additionally, infants born to mothers in the At-Risk + Polyhydramnios were $174 \%$ more likely to require a NICU admission compared to those born to mothers in the Normal + Normal Fluid group.

There were no differences between the groups in terms of average base excess or presence of umbilical artery $\mathrm{pH}<7.1$. (Table 3, Supplemental Table 2, Supplemental Table 3). However, the average birthweight among infants born to mothers in the Normal + Normal Fluid was much less compared to those born to mothers in the Normal + 
Table I Descriptive Statistics

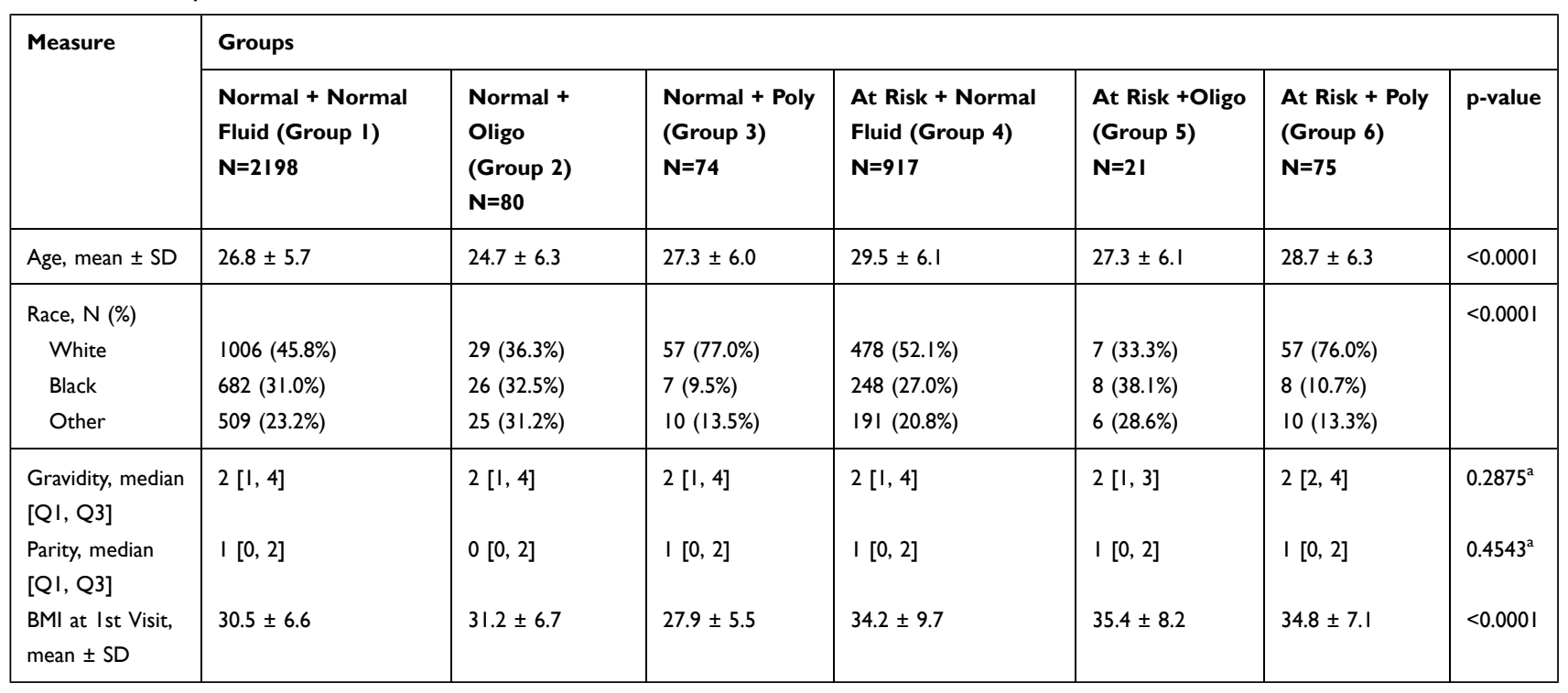

Note: ${ }^{a}$ Denote statistical test using Kruskal-Wallis.

Table 2 Association Between Maternal Groups and Categorical Infant Outcomes (Adjusted)

\begin{tabular}{|l|l|l|l|l|l|l|l|l|l|l|}
\hline Group & Amnioinfusion & p-value & $\begin{array}{l}\text { Variables } \\
\text { Influencing } \\
\text { Delivery }\end{array}$ & p-value & $\begin{array}{l}\text { Lates } \\
\text { Influencing } \\
\text { Delivery }\end{array}$ & p-value & Meconium & p-value & $\begin{array}{l}\text { NICU } \\
\text { Admission }\end{array}$ & p-value \\
\hline 1 & Ref & 0.143 & Ref & 0.076 & Ref & 0.060 & Ref & 0.395 & Ref \\
2 & $1.83(0.64,5.22)$ & & $0.86(0.45,1.64)$ & & $2.06(1.14,3.73)$ & & $1.51(0.76,2.99)$ & & $1.69(0.81,3.55)$ & \\
3 & $0.86(0.20,3.59)$ & & $1.08(0.60,1.94)$ & & $1.04(0.47,2.33)$ & & $1.42(0.69,2.92)$ & & $1.65(0.85,3.23)$ \\
4 & $1.69(1.12,2.55)$ & & $1.33(1.09,1.63)$ & & $1.33(1.04,1.70)$ & & $0.82(0.61,1.10)$ & & $2.06(1.63,2.60)$ \\
5 & $1.92(0.25,14.6)$ & & $1.27(0.42,3.82)$ & & $1.93(0.63,5.87)$ & & $0.50(0.07,3.79)$ & & $2.11(0.65,6.86)$ \\
6 & $0.46(0.06,3.39)$ & & $0.71(0.36,1.39)$ & & $1.08(0.51,2.29)$ & & $1.18(0.56,2.52)$ & & $2.74(1.54,4.87)$ & \\
\hline
\end{tabular}

Notes: Groups I (Normal+Normal Fluid); 2 (Normal+Oligo Fluid); 3 (Normal+Poly Fluid); 4 (At-Risk+Normal Fluid); 5 (At-Risk+Oligo Fluid); 6 (At-Risk+Poly Fluid); Analyses adjusted for maternal age, race, parity, gravidity, BMI at first visit, estimated gestational age at last assessment.

Polyhydramnios and At-Risk + Polyhydramnios groups, with a least squares mean difference of 345.77 grams $(95 \% \mathrm{CI}$ : 214.6, 476.9) and 507.87 grams (95\% CI: $378.3,637.5)$, respectively (Table 3 ). In contrast, mothers in the Normal + Normal Fluid group delivered infants with greater birthweight compared to those in both the At-Risk + Normal Fluid and At-Risk + Oligohydramnios groups with least squares mean difference of -121.68 grams (95\% CI: $-167.1,-76.3)$ and -513.3 grams (95\% CI: $-754.0,-272.6)$. (Table 3).

\section{Discussion}

\section{Principal Findings}

Our study looked at six unique groups with a Normal pregnancy with Normal fluid group as the reference group and the impact of AFVs on adverse pregnancy outcomes. There were no significant differences in many of our selected markers of adverse pregnancy outcomes (late decelerations or variable decelerations influencing delivery, need for amnioinfusion, umbilical artery $\mathrm{pH}<7.1$ and meconium staining) with respect to risk stratification or amniotic fluid volume. The only clinically significant differences we found in these groups were incidence of NICU admission and birthweight.

\section{Results}

NICU admission is an important marker of neonatal morbidity. NICU admission was found to be increased in the At-Risk group with Normal Fluid compared to the Normal group with Normal Fluid. The highest risk of NICU admission was in the At-Risk group with polyhydramnios. A prospective study from 2010 noted that the presence of polyhydramnios was associated with NICU admission, abnormal fetal heart rate tracing, and increased birthweight. ${ }^{15}$ The increased birthweight noted in our study in women with polyhydramnios (in both normal 
Table 3 Association Between Maternal Groups and Continuous Infant Outcomes (Adjusted)

\begin{tabular}{|l|l|l|l|l|l|l|}
\hline \multirow{2}{*}{ Group } & \multicolumn{4}{l}{ Least Squares Means Difference with Group I as the Reference Group } & \multicolumn{2}{l|}{} \\
\cline { 2 - 7 } & Birthweight & p-value $^{\mathbf{a}}$ & Umbilical Artery pH & p-value $^{\mathbf{a}}$ & Bases Excess $^{\text {p-value }}$ \\
\hline 2 vs I & $-92.39(-218.0,33.22)$ & $<0.0001$ & $-0.001(-0.019,0.017)$ & 0.148 & $-0.121(-0.762,0.519)$ & 0.757 \\
3 vs I & $345.77(214.6,476.9)$ & & $-0.026(-0.055,0.004)$ & & $0.390(-0.682,1.461)$ \\
4 vs I & $-121.68(-167.1,-76.3)$ & & $-0.008(-0.016,-0.001)$ & & $0.027(-0.249,0.304)$ \\
5 vs I & $-513.30(-754.0,-272.6)$ & & $-0.017(-0.051,0.017)$ & & $0.695(-0.552,1.942)$ \\
6 vs I & $507.87(378.3,637.5)$ & & $-0.007(-0.033,0.019)$ & & $0.438(-0.515,1.391)$ \\
\hline
\end{tabular}

Notes: Groups I (Normal+Normal Fluid); 2 (Normal+Oligo Fluid); 3 (Normal+Poly Fluid); 4 (At-Risk+Normal Fluid); 5 (At-Risk+Oligo Fluid); 6 (At-Risk+Poly Fluid); a'Denote overall F-test p-value; Analyses adjusted for maternal age, race, parity, gravidity, BMI at first visit, estimated gestational age at last assessment.

and at-risk pregnancies) has been confirmed in a prior systematic review and meta-analysis. ${ }^{16}$ Our study also showed that patients with at-risk pregnancies with both normal fluid and oligohydramnios had smaller fetuses than those patients with normal pregnancies and normal fluid. Additionally, normal pregnancies with oligohydramnios had larger fetuses than at-risk pregnancies with oligohydramnios. Specifically, a 400 gram difference in the at-risk pregnancies with abnormal fluid and the normal pregnancies with abnormal fluid is clinically significant.

\section{Clinical Implications}

Amniotic fluid assessment has been in use for over 30 years for the prediction of adverse neonatal outcomes. ${ }^{8}$ Sonographic estimation correlates with direct measurement of amniotic fluid volumes. ${ }^{17,18}$ A recent retrospective study looked at two standard nomograms for amniotic fluid indices and adverse perinatal outcomes and found that mildly abnormal sonographically estimated amniotic fluid did not have a significant impact on the risk of adverse pregnancy outcomes. ${ }^{18}$ Another recent study of direct and estimated amniotic fluid measurement showed that patients with isolated oligohydramnios without other comorbidities were not associated with adverse pregnancy outcome. ${ }^{19}$ Magann et al studied actual amniotic fluid volumes, ultrasonographically assessed amniotic fluid volumes, and neonatal outcomes in a prior prospective study, and found that amniotic fluid volume was not predictive of adverse outcomes at the time of delivery. ${ }^{20}$

\section{Research Implications}

There is a great need in modern obstetric practice to further refine risk stratification and amniotic fluid volumes to continue to improve pregnancy outcome without increasing unnecessary interventions. A large retrospective study of high risk and low risk pregnancies and amniotic fluid volume from 2005 also found that amniotic fluid abnormalities were not strongly associated with adverse pregnancy outcomes. $^{21}$ It may be that the increased risk of morbidity lies, not at the extremes of normal amniotic fluid volume, but at the extremes of abnormal amniotic fluid volume. For example, in our study, we only looked at abnormal SDP for oligohydramnios and abnormal AFI for polyhydramnios. It may be that patients with both abnormal SDP and AFI could be at higher risk for adverse outcomes than those with just one abnormal measurement. It also could be that the current cut-offs for abnormal SDP and AFI are not extreme enough.

Additionally, much of the literature concerning oligohydramnios has involved preterm prelabor of the membranes (PPROM). Within this group, lower amniotic fluid volume is associated with increased risk of neonatal sepsis and neonatal death. $^{22}$ In another retrospective study of PPROM, severe oligohydramnios (defined by the authors as AFI $<3 \mathrm{~cm}$ ) was associated with increased risk of APGAR scores less than 7 at 1 minute, neonatal sepsis, and early neonatal mortality. ${ }^{23}$ Within the realm of PPROM, extremely low amniotic fluid volume is associated with worse outcomes.

\section{Strengths and Limitations}

The limitations of our study include the retrospective nature and the small sample of patients with abnormal amniotic fluid volumes which may have limited our power to detect rare outcomes. The strengths of our study are the multi-centered collection sites and the large sample of patients overall. It is also a strength of our study that patients with PPROM were excluded from the study.

\section{Conclusions}

Amniotic fluid assessment remains an important means of assessing fetal well-being. Given the daily use of the sonographic estimate of AFV in obstetric practice, it is important to continue to study and define abnormal volumes. Our study attempted to 
further define risk of adverse pregnancy outcomes by defining the pregnancy as normal or at-risk and by stratifying the pregnancies based on AFVs. We did not find increased risk of many adverse perinatal outcomes amongst at-risk pregnancies with abnormal fluid as anticipated; however, NICU admission was more frequent amongst pregnancies with polyhydramnios. More multi-center, high-quality studies should be designed and conducted to further elucidate the relationship between pregnancy risk, amniotic fluid, and adverse outcomes, as this could influence practice patterns for women's health care providers.

\section{Acknowledgments}

We would like to thank Donna Eastham, BS, CRS for her assistance in editing and submitting this manuscript. JRW and SME are military service members. The views expressed in this article reflect the results of research conducted by the authors and do not necessarily reflect the official policy or position of the Department of the Army, Department of the Navy, Department of Defense, or the United States Government.

\section{Disclosure}

Everett F. Magann is the co-author of the Assessment of Amniotic Fluid Volume Physiology on UpToDate and receives royalties for this work. The authors report no other potential conflicts of interest for this work.

\section{References}

1. Dubil EA, Magann EF. Amniotic fluid as a vital sign for fetal wellbeing. Australas J Ultrasound Med. 2013;16(2):62-70. doi:10.1002/j.2205-0140.2013.tb00167.x

2. Gagnon R, Harding R, Brace RA. Amniotic fluid and fetal urinary responses to severe placental insufficiency in sheep. Am J Obstet Gynecol. 2002;186(5):1076-1084. doi:10.1067/mob.2002.122291

3. Phelan JP, Park YW, Ahn MO, Rutherford SE. Polyhydramnios and perinatal outcome. J Perinatol. 1990;10(4):347-350.

4. Moore TR. The role of amniotic fluid assessment in evaluating fetal well-being. Clin Perinatol. 2011;38(1):33-46. doi:10.1016/j. clp.2010.12.005

5. Moore TR, Cayle JE. The amniotic fluid index in normal human pregnancy. Am $J$ Obstet Gynecol. 1990;162(5):1168-1173. doi:10.1016/0002-9378(90)90009-v.

6. Owen J, Albert PS, Buck Louis GM, et al. A contemporary amniotic fluid volume chart for the United States: the NICHD fetal growth studies-singletons. Am J Obstet Gynecol. 2019;221(1):67.e1-.e12. doi:10.1016/j.ajog.2019.02.030.

7. Peixoto AB, Caldas TM, Martins WP, Da Silva Costa F, Araujo Junior E. Unconditional reference values for the amniotic fluid index measurement between $26 \mathrm{w} 0 \mathrm{~d}$ and $41 \mathrm{w} 6 \mathrm{~d}$ of gestation in low-risk pregnancies. J Matern Fetal Neonatal Med. 2016;29(20):3243-3248. doi:10.3109/14767058.2015.1123246.

8. Chamberlain PF, Manning FA, Morrison I, Harman CR, Lange IR. Ultrasound evaluation of amniotic fluid volume. I. The relationship of marginal and decreased amniotic fluid volumes to perinatal outcome. Am J Obstet Gynecol. 1984;150(3):245-249. doi:10.1016/s00029378(84)90359-4.
9. von Elm E, Altman DG, Egger M, Pocock SJ, Gotzsche PC, Vandenbroucke JP. The strengthening the reporting of observational studies in epidemiology (STROBE) statement: guidelines for reporting observational studies. Lancet. 2007;370(9596):1453-1457. doi:10.1016/s0140-6736(07)61602-x.

10. Nabhan AF, Abdelmoula YA. Amniotic fluid index versus single deepest vertical pocket as a screening test for preventing adverse pregnancy outcome. Cochrane Database Syst Rev. 2008;(3): Cd006593. doi:10.1002/14651858.CD006593.pub2.

11. Kehl S, Schelkle A, Thomas A, et al. Single deepest vertical pocket or amniotic fluid index as evaluation test for predicting adverse pregnancy outcome (SAFE trial): a multicenter, open-label, randomized controlled trial. Ultrasound Obstet Gynecol. 2016;47 (6):674-679. doi:10.1002/uog.14924.

12. Rosati P, Guariglia L, Cavaliere AF, et al. A comparison between amniotic fluid index and the single deepest vertical pocket technique in predicting adverse outcome in prolonged pregnancy. $J$ Prenat Med. 2015;9(1-2):12-15. doi:10.11138/jpm/2015.9.1.012.

13. Hughes DS, Magann EF, Whittington JR, Wendel MP, Sandlin AT, Ounpraseuth ST. Accuracy of the ultrasound estimate of the amniotic fluid volume (amniotic fluid index and single deepest pocket) to identify actual low, normal, and high amniotic fluid volumes as determined by quantile regression. J Ultrasound Med. 2020;39 (2):373-378. doi:10.1002/jum.15116.

14. Helwig JT, Parer JT, Kilpatrick SJ, Laros RK. Umbilical cord blood acid-base state: what is normal? Am J Obstet Gynecol. 1996;174 (6):1807-12; discussion 12-4. doi:10.1016/s0002-9378(96)70214-4.

15. Magann EF, Doherty DA, Lutgendorf MA, Magann MI, Chauhan SP, Morrison JC. Peripartum outcomes of high-risk pregnancies complicated by oligo- and polyhydramnios: a prospective longitudinal study. J Obstet Gynaecol Res. 2010;36(2):268-277. doi:10.1111/j.14470756.2009.01145.x.

16. Morris RK, Meller CH, Tamblyn J, et al. Association and prediction of amniotic fluid measurements for adverse pregnancy outcome: systematic review and meta-analysis. BJOG. 2014;121(6):686-699. doi:10.1111/1471-0528.12589.

17. Magann EF, Nolan TE, Hess LW, Martin RW, Whitworth NS, Morrison JC. Measurement of amniotic fluid volume: accuracy of ultrasonography techniques. Am J Obstet Gynecol. 1992;167 (6):1533-1537. doi:10.1016/0002-9378(92)91734-r.

18. Krispin E, Berezowsky A, Chen R, et al. Updating the amniotic fluid index nomograms according to perinatal outcome. J Matern Fetal Neonatal Med. 2020;33(1):113-119. doi:10.1080/14767058.2018.1487936.

19. Madendag Y, Madendag IC, Sahin E, Aydin E, Sahin ME, Acmaz G. How well do the popular ultrasonic techniques estimate amniotic fluid volume and diagnose oligohydramnios, in fact? Ultrasound $Q$. 2019;35(1):35-38. doi:10.1097/ruq.0000000000000408.

20. Magann EF, Chauhan SP, Doherty DA, Barrilleaux PS, Martin JN, Morrison JC. Predictability of intrapartum and neonatal outcomes with the amniotic fluid volume distribution: a reassessment using the amniotic fluid index, single deepest pocket, and a dye-determined amniotic fluid volume. Am J Obstet Gynecol. 2003;188(6):1523-7; discussion 7-8. doi:10.1067/mob.2003.381.

21. Ott WJ. Reevaluation of the relationship between amniotic fluid volume and perinatal outcome. Am J Obstet Gynecol. 2005;192(6):1803-9; discussion 9. doi:10.1016/j.ajog.2004.12.062.

22. Mousavi AS, Hashemi N, Kashanian M, Sheikhansari N, Bordbar A, Parashi S. Comparison between maternal and neonatal outcome of PPROM in the cases of amniotic fluid index (AFI) of more and less than $5 \mathrm{~cm}$. J Obstet Gynaecol. 2018;38(5):611-615. doi:10.1080/ 01443615.2017.1394280.

23. Souza AS, Patriota AF, Guerra GV, Melo BC. Evaluation of perinatal outcomes in pregnant women with preterm premature rupture of membranes. Rev Assoc Med Bras. 2016;62(3):269-275. doi:10.1590/1806-9282.62.03.269. 


\section{Publish your work in this journal}

The International Journal of Women's Health is an international, peerreviewed open-access journal publishing original research, reports, editorials, reviews and commentaries on all aspects of women's healthcare including gynecology, obstetrics, and breast cancer. The

manuscript management system is completely online and includes a very quick and fair peer-review system, which is all easy to use. Visit http://www.dovepress.com/testimonials.php to read real quotes from published authors

Submit your manuscript here: https://www.dovepress.com/international-journal-of-womens-health-journal 